\title{
Anabases
}

ANABASES Traditions et réceptions de l'Antiquité

4 | 2006

Varia

\section{Les mercenaires de Grèce et d'ailleurs dans le DAGR}

\section{Sandra Péré-Noguès}

\section{OpenEdition}

\section{Journals}

Édition électronique

URL : http://journals.openedition.org/anabases/3004

DOI : 10.4000/anabases.3004

ISSN : 2256-9421

\section{Éditeur}

E.R.A.S.M.E.

\section{Édition imprimée}

Date de publication : 1 octobre 2006

Pagination : 237-242

ISSN : 1774-4296

\section{Référence électronique}

Sandra Péré-Noguès, "Les mercenaires de Grèce et d'ailleurs dans le DAGR », Anabases [En ligne], 4 I 2006, mis en ligne le 01 janvier 2012, consulté le 20 octobre 2019. URL : http://

journals.openedition.org/anabases/3004; DOI : 10.4000/anabases.3004 


\section{Les mercenaires de Grèce et d'ailleurs dans le $D A G R$}

SANDra PÉRÉ-Noguès

L'

ARTIClE CONSACRÉ AUX MERCENAIRES ("Mercenarii ») se trouve dans la seconde partie du tome III du Dictionnaire des Antiquités Grecques et Romaines. Publié en 1904, il en occupe dix-huit pages de texte et contient cinq illustrations. Son rédacteur est Albert Martin qui fit paraître en 1886 une étude sur les cavaliers athéniens ${ }^{1}$.

L'article s'articule grosso modo autour de deux parties la Grèce et Rome, la première étant beaucoup plus fournie que la seconde. Nous limiterons ici notre réflexion à la Grèce, plus particulièrement à l'époque archaïque et classique. Il faut souligner la qualité des sources documentaires, aussi bien littéraires qu'archéologiques et épigraphiques. Sur le plan iconographique, la moisson est plus mince et plutôt en lien avec l'image du soldat ou du guerrier que du mercenaire ${ }^{2}$. Les deux illustrations soulèvent aussi des problèmes de localisation, l'aryballe dit du Louvre n'étant plus dans ce musée ${ }^{3}$. L'approche est également orientée puisque les mercenaires sont ici perçus à travers les puissances qui les ont recrutés. La démarche, qui s'inscrit dans le courant positiviste, explique l'extrême fidélité aux textes littéraires ${ }^{4}$. Notons que cette approche

1 A.H. Martin, Les Cavaliers Athéniens, Paris, 1886.

2 Pour les illustrations il s'agit des numéros 4928 et 4929, représentant pour l'une la statuette d'un mercenaire grec trouvée à Chypre, pour l'autre un aryballe du Louvre qui reproduit la tête d'un mercenaire grec.

3 Nous remercions M. François Lissarrague pour ce renseignement.

4 Pour la même approche positiviste, voir : H.W. PARKE, Greek mercenary soldiers from the earliest times to the battle of Ipsus, Chicago, 1933 ; G.T. GRIFFITH, The mercenaries of the Hellenistic World, Chicago, 1935. 
traditionnelle a perduré jusqu'aux travaux de Marcel Launey ${ }^{5}$ en 1949 sur les armées hellénistiques mais qu'elle est aujourd'hui abandonnée. Les recherches ${ }^{6}$ amorcées dans les années 60 et 70 ont totalement renouvelé notre approche du problème des mercenaires et en premier lieu celui de la définition du mercenaire.

Albert Martin donne au tout début de son article sa propre interprétation de la condition de mercenaire : "La condition de mercenaire ou de soldat étranger dont on paye le service suppose une organisation sociale et politique particulières, c'est-à-dire que, sur ce point, comme sur bien d'autres, il y a un rapport étroit entre la constitution politique d'un État et l'organisation de ses forces militaires ». L'auteur ajoute également une liste de termes grecs qui font selon lui directement référence aux mercenaires: misthophoros, epikouroi, xénoi, stratiôtai... Sur cette liste quelques précisions sont nécessaires. Si le misthophoros est "celui qui reçoit une solde " le xénos est l'étranger. Plus ambigu est le recours au terme d'épikouros, qui signifie allié. Dans un article assez récent, F. Landucci Gattinoni 7 note que le vocabulaire employé pour l'époque archaïque, et notamment chez Hérodote, ressort de la mentalité aristocratique, ce qui expliquerait pourquoi le mercenaire est traité comme un allié et non comme un soldat payé pour ses services. La rupture terminologique intervient avec Thucydide qui introduit une dimension plus économique et sociale dans la définition du mercenaire. Quant au terme stratiôtès, il définit à partir du IVe siècle le "soldat de métier ".

Si l'on examine les définitions modernes, les variations sont sensibles d'un auteur à l'autre mais mettent en exergue trois particularités qui peuvent définir le statut du

5 M. LAUneY, Recherches sur les armées hellénistiques, 2 vol., Paris, 1949-1950 (nouvelle édition, Paris, 1987, avec addenda et suppléments bibliographiques dans la postface de Y. Garlan, Ph. Gauthier et Cl. Orrieux)

6 Outre celle de M. Launey qui reste une référence, voir : A. AYMARD, “ Mercenariat et histoire grecque ", dans Etudes d'Histoire ancienne, 1967, p. 487-498 ; Y. GARLAN, La Guerre dans l'Antiquité, Paris, 1972 ; Y. GARLAN, Guerre et économie en Grèce ancienne, Paris, 1989 ; P. DUCREY, Guerre et guerriers dans la Grèce antique, Paris, 1985 ; L. MARINOVIC, Le mercenariat grec et la crise de la polis, Paris, 1988 ; M. BETTALLI, I mercenari nel mondo greco. I- Dalle origini alla fine del V sec. a. C., Pise, 1995. Cette liste n'est pas exhaustive car les travaux se sont multipliés dans les dernières années : J.-Ch. Couvenhnes et S. PÉré-NoguÈS, " Quoi de neuf sur la guerre ? Perspectives de recherche et données nouvelles sur la guerre dans le monde méditerranéen aux époques archaïque, classique et hellénistique (à partir de quelques publications) ”, Pallas, 2005, p. 379-399.

7 F. LANDUCCI GATTINONI, “I mercenari e l'ideologia della guerra ”, dans Il pensiero sulla guerra nel mondo antico, éd. M. Sordi, Milan, 2001, p. 65-85. 
mercenaire : le professionnalisme, la condition de "salarié " (rémunéré pour ses services), et le statut d'étranger ${ }^{8}$.

Un aspect n'est pas non plus mis en exergue par A. Martin du fait de son approche positiviste : il s'agit de l'émergence du mercenariat à partir de la guerre du Péloponnèse. Le concept de mercenariat est à vrai dire récent puisqu'il est utilisé pour la première fois par A. Aymard par analogie avec "salariat 9 ". Il est important de souligner que ce concept a l'avantage d'établir un saut quantitatif majeur dans l'histoire des mercenaires, puisque c'est à partir de cette époque que leur nombre est désormais suffisamment important pour avoir des répercussions directes sur les cités. Albert Martin parâtt sensible à cet accroissement du nombre de mercenaires, mais il n'en mesure surtout que l'impact politique et militaire, laissant de côté tous les aspects économiques et sociaux.

Nous voudrions maintenant commencer par reprendre le travail accompli par Albert Martin et voir comment peut se penser une mise à jour, étant donné que l'objectif est à long terme d'en proposer une révision complète. Nous avons donc porté notre attention sur deux questions abordées au début de l'article, que nous ne traiterons pas entièrement mais sur lesquelles nous voudrions faire quelques commentaires.

Tout d'abord il faut évoquer le problème des Cariens qu'Albert Martin présente ainsi : "Les Cariens sont les premiers des Grecs, dit Ephore, qui se sont engagés comme mercenaires. C'était une race militaire. Hérodote leur attribue l'invention du cimier à casque et des boucliers à poignée. Archiloque fait du nom de Carien le synonyme de mercenaire. Dès les temps les plus anciens, on trouve les Cariens et les Crétois au service des monarchies orientales : ce sont les Kreti-Pelti des rois juifs ; Gygès, Crésus, tous les rois de la Lydie ont des mercenaires, qui sont en grande partie Cariens ${ }^{10}$."

8 Ainsi, M. Launey (op. cit., p. 26) considérait que «l'individu qui prend du service auprès d'une armée autre que celle de sa patrie est généralement engagé comme mercenaire ». Quelques années plus tard, André Aymard (op. cit., p. 487) le définit comme " un soldat de métier [qui] assure par l'exercice de sa profession son existence matérielle » et qui sert dans une armée dans laquelle il est un étranger. La définition la plus globale et sans doute la plus juste est celle qu'a proposée Y. Garlan (op. cit., p. 67) : «Un soldat professionnel dont la conduite est avant tout dictée, non pas par son appartenance à une communauté politique, mais par l'appât du gain. " Dernière en date est la définition de M. Bettalli ( $o p$. cit., p. 23) : celui qui " combat au service d'un pays qui n'est pas le sien, hors de toute logique d'alliance, de relations, de traités, avec l'unique objectif de percevoir un salaire et d'améliorer sa condition personnelle ».

10 A. MARTIN, op. cit., p. 1784. 
L'auteur reproduit dans son intégralité la tradition historiographique sur les Cariens : à la fois innovateurs dans l'armement et premiers mercenaires. Cette tradition historiographique est surtout véhiculée par Hérodote à la suite de témoignages plus anciens comme ceux d'Alcée ou d'Anacréon. L'historien d'Halicarnasse laissait entendre que les Cariens inventèrent le bouclier hoplitique, mais sur le plan archéologique la relation entre cette innovation technologique et eux n'est pas du tout établie comme l'a montré A.M. Snodgrass ${ }^{11}$. En effet, si les premiers hoplites semblent apparaître dans le Péloponnèse, la région qui a fourni le modèle de leur nouvel équipement reste énigmatique ${ }^{12}$. Le débat est encore ouvert non seulement sur les origines du bouclier mais aussi sur celles d'autres armes. Par ailleurs, la référence concernant leur recrutement auprès des rois juifs qui remonterait à la fin du IXe siècle est sérieusement mise en doute car elle se fonde sur deux termes hébreux Kerêtîm et Pelêtî̀m qui désignent des personnes respectivement originaires de Crète et des Philistins ${ }^{13}$. Néanmoins, aucun doute n'existe sur le fait que les Cariens furent très tôt des mercenaires en raison de la pauvreté de leur territoire. Du reste, un proverbe très connu sur les Cariens et leur sacrifice comme mercenaires à la place des Grecs circulait à la fin du ve siècle et se retrouve dans de nombreuses œuvres 14 .

Le second problème est celui des mercenaires grecs en Égypte. L'analyse réalisée par A. Martin est tout à fait originale pour son époque, car elle s'appuie sur un dossier documentaire très bien fourni et rassemblant aussi bien des sources textuelles qu'épigraphiques ou archéologiques, ces dernières étant souvent assez récentes. Reprenons les pièces du dossier pour voir comment les aborder aujourd'hui.

La plus ancienne attestation d'un établissement de Grecs en Égypte est celle des Milésiens " qui fondèrent dans la seconde moitié du VIII siècle le teichos Milèsion entre la bouche Bolbitine et la bouche Sébennytique 15 \%. Mais elle prête à discussion dans la mesure où elle n'est transmise que par des sources largement postérieures comme Strabon ou Eusèbe de Césarée. Le terrain devient plus sûr à partir du siècle suivant, notamment du règne de Psammétique Ier à partir de 664.

On constate d'abord que ce recours aux mercenaires grecs s'est inscrit dans la durée puisqu'il couvre plus d'un siècle (664-526). L'histoire des relations entre Grecs et souverains saïtes fut complexe : à des moments d'acceptation volontaire et encouragée (sous les règnes de Psammétique Ier ou Apriès) ont succédé des mouvements de rejet contre ces étrangers dont la puissance royale ne pouvait se passer. L'arrivée de ces " hommes de bronze " a eu également deux prolongements majeurs : l'introduction

11 A. SNODGRASS, "Carian armourers : the growth of a tradition ", Journal of Hellenic Studies, 1964, 84, p. 107-118.

14 M. BETTALLI, op. cit., p. 110-111.

15 A. MARTIN, op. cit., p. 1785. 
d'un équipement militaire inconnu des usages égyptiens et la résidence accordée à des étrangers, de surcroît des soldats. La création de Stratopéda évoquée par Hérodote et Diodore 16 a en effet été confirmée sur le terrain par les fouilles de deux sites : celui de Tell Defenneh sur la branche pélusienne du Delta du Nil où on a retrouvé de la céramique grecque et des armes grecques. Ce site, auquel A. Martin fait référence, fut fouillé par Flinders Petrie en 1885 et 1886 ; implanté à proximité d'une ville de 20000 habitants où vivaient des marchands du Proche-Orient, il est situé sur la route caravanière entre l'Égypte et la Syrie. Un autre site qui fut fouillé dans les années 1980 est celui de Migdol à vingt kilomètres au nord du précédent et sur l'autre rive du Nil 17 . Sur le plan archéologique, il est bien évident que nos connaissances ont évolué depuis la rédaction de l'article et qu'une mise à jour s'impose. Mais il faut encore reconnaître l'intérêt porté par Albert Martin à l'archéologie, intérêt souvent peu marqué à son époque par les spécialistes de la guerre.

Plus tard les Grecs furent déplacés à Memphis où ils furent cantonnés dans des quartiers grecs puis à Naucratis. De fait, il existe un quartier à la périphérie de Memphis, le Karikon, dont la nécropole a livré le groupe le plus notable d'inscriptions cariennes ${ }^{18}$. Ces diverses installations amènent évidemment à s'interroger sur l'intégration des Grecs dans la société égyptienne, notamment sur la question des mariages mixtes. Ces débats n'apparaissent pas dans l'article d'Albert Martin dont l'approche est très représentative de la mentalité de l'époque ${ }^{19}$.

D'autres documents importants sont aussi à prendre en compte : d'une part les inscriptions d'Abu Simbel, d'autre part l'inscription du mercenaire Pédon aujourd'hui perdue. Les inscriptions gravées sur le pied gauche de la statue colossale de Ramsès II à Abu Simbel sont signalées par A. Martin et restent aujourd'hui l'un des témoignages les

16 Hérodote II, 152 : «Aux Ioniens et aux Cariens qui l'avaient secondé, Psammétique donna des terres où s'établir, situées en vis-à-vis de chaque côté du Nil ; on appelle cet endroit les Camps (Stratopeda). Il leur donna ce territoire et s'acquitta également de toutes ses autres promesses. Il leur confia aussi des jeunes Égyptiens à qui enseigner la langue grecque; ces jeunes gens qui apprirent notre langue sont les ancêtres des interprètes qu'on trouve aujourd'hui en Égypte. Les Ioniens et les Cariens habitèrent longtemps ce territoire ; il se trouve près de la mer, un peu au-dessous de Bubastis, sur la bouche du Nil qu'on appelle Pélusienne. Plus tard le roi Amasis les fit venir à Memphis, quand il les prit comme gardes du corps au lieu d'Égyptiens. C'est depuis leur installation en Egypte et grâce à leurs relations avec la Grèce que nous avons, nous autres, une connaissance exacte de l'histoire de l'Égypte, à partir du roi Psammétique ; car ils ont été les premiers hommes parlant une langue étrangère à s'établir en Égypte. Dans la région qu'ils ont quittée, on voyait encore de mon temps les hangars de leurs navires et les ruines de leurs habitations. » Voir aussi Diodore de Sicile I, 67.

17 Sur Tell Defenneh et Migdol : M. BetTalli, op. cit., p. 63-64. D’autres sites ont été fouillés en Israël.

19 Voir A. MarTin, op. cit., p. 1787. 
plus éloquents de la présence mercenaire en Égypte ${ }^{20}$. Elles sont probablement datées de 591 et ont l'avantage de fournir quelques renseignements sur l'organisation de l'armée égyptienne et l'intégration des troupes grecques, mais aussi sur l'origine et le degré d'alphabétisation des mercenaires eux-mêmes. Quant à l'inscription de Pédon gravée en alphabet ionien archä̈que sur une statue cube de type égyptien, elle fut découverte près de Priène ${ }^{21}$ dans les années 1980 . Il s'agit sans doute d'un mercenaire rentré dans sa patrie à la fin du règne de Psammétique Ier. Cette inscription serait évidemment à ajouter au corpus de documents sur la question des mercenaires grecs en Égypte.

Ce bref état des lieux aura montré tout l'intérêt de proposer aujourd'hui une version renouvelée de l'article, compte tenu des limites de l'approche adoptée par Albert Martin et du renouvellement de nos informations sur ces sujets. Il faut ajouter que d'autres axes d'étude sont aujourd'hui privilégiés et n’apparaissent pas dans l'article. En effet, depuis la parution en 1994 de l'étude de G. Tagliamonte 22 sur les mercenaires italiques, les études se sont multipliées privilégiant désormais une ouverture géographique nouvelle : l'horizon n'est plus seulement limité à l'espace égéen mais il déborde largement sur le monde occidental grec et non grec. De fait cette pratique du mercenariat était largement partagée par d'autres peuples dits barbares, Celtes, Ibères, Campaniens ou Baléares. C'est dans cette perspective élargie que les mercenaires et le mercenariat doivent aujourd'hui être envisagés et c'est aussi dans cette perspective que nous souhaiterions réactualiser l'article d'Albert Martin.

\begin{tabular}{l} 
SANDRA PÉRÉ-NOGUÈS \\
\hline Université Toulouse II, UTAH UMR 5608 \\
5 allées A. Machado \\
31058 - Toulouse cedex 9 \\
sandra.perenogues@univ-tlse2.fr
\end{tabular}

20 Voir pour une reprise du dossier : M. BETTALli, op. cit., p. 66-69.

21 C. Ampolo et E. Bresciani, "Psammetico re d'Egitto e il mercenario Pedon ", Egitto e Vicino Oriente, 11 (1988), p. 237-253.

22 G. Tagliamonte, I Figli di Marte. Mobilità, mercenari e mercenariato italici in Magna Grecia e Sicilia, Rome, 1994. 